\title{
Application of an ecosystem-based spatial management approach in a coastal area in western Greece
}

\author{
V. Vassilopoulou ${ }^{1}$, Y. Issaris ${ }^{1}$, S. Giakoumi ${ }^{1}$, G. Mavromati ${ }^{2}$, \\ M. Pantazi ${ }^{1}$, A. Kokkali ${ }^{1}$, S. Kavvadas ${ }^{1}$, I. Maina ${ }^{1}$, \\ A. Dogrammatzi ${ }^{1}$, C. Anagnostou ${ }^{1}$, S. Katsanevakis ${ }^{3}$ \\ \& P. Panayotidis ${ }^{1}$ \\ ${ }^{1}$ Hellenic Centre for Marine Research, Greece \\ ${ }^{2}$ Center for Water Sciences, Michigan State University, USA \\ ${ }^{3}$ Institute for Environment and Sustainability, JRC, Italy
}

\begin{abstract}
Coastal areas in the EU are usually subjected to various anthropogenic pressures, with growing conflicts among economic activities. The FP7 project MESMA has developed a flexible framework to monitor and evaluate Spatially Managed Areas (SMAs) in both coastal and offshore waters and has tested it in nine case studies, one of which is a region in western Greece, including the Inner Ionian Archipelago and the adjacent gulfs. One of the first steps of the approach is to provide visualization of the main ecosystem components and human activities /pressures, on the basis of existing spatial information and expert judgment, addressing also issues related to data uncertainty. GIS tools were used for mapping ecosystem components, and main human activities. As substantial overlapping was identified between ecological features and human pressures, an effort was made to apply the principles of systematic conservation planning using the decision making tool Marxan, in order to propose scenarios aiming to contribute to the sustainable management of the area under study. Interaction with key stakeholders coming from various action arenas revealed the need for developing and enforcing more coherent and transparent strategies engaging endusers in the process.
\end{abstract}

Keywords: marine spatial planning, integrated framework, ecosystem components, human activities, conflicts. 


\section{Introduction}

In recent years, conflicting issues between environmental conservation and human activities in the marine environment grow and integrated Marine Spatial Planning (MSP) has been identified as a promising tool that considers the existing patterns of human activities conflicting either among them, and/or with conservation features, and proposes mitigation of conflicts through adaptive management. MSP has been introduced as a management approach in the general context of nature conservation originating from the Great Barrier Reef Marine Park over thirty years ago. Implementation of a MSP framework requires a multifaceted approach, which starts with the establishment of a solid scientific basis of the problem to be tackled, and followed by integrated analysis of all factors interplaying, i.e. political framework, economical state, human conflicts, and environmental protection. A management plan for the study site is needed, but it should be developed in a holistic manner, targeting at protection, restoration and sustainable development of the environment.

An ongoing FP7 project called "MESMA" (Monitoring and Evaluation of Spatially Managed Areas (SMAs)) has developed a seven-step framework that has been tested for monitoring and evaluation of SMAs on several European marine regions [1]. This study presents outcomes from the application of a MSP framework created in the frame of the MESMA project on the Greek case study, where conflicts between conservation and human activities (mainly fisheries and tourism) are intense. This attempt aims to contribute to recent efforts on initializing MSP in the country focusing particularly on the evaluation of existing management measures to meeting operational objectives linked mainly to high level policy goals.

\section{Methods}

\subsection{The MESMA framework}

\subsubsection{Ground disciplines and general information}

MESMA aims at developing strategies, guidelines, tools and a systematic framework to facilitate integrated monitoring, evaluation and implementation of Spatially Managed Areas (SMAs). The central part of the MESMA spatial management toolbox is a generic and flexible framework that describes a best practice process of how to assess a given spatial management within a discrete marine area with clearly defined boundaries in space and time.

The MESMA framework is applicable at any spatial scale independent from the natural and socioeconomic factors relevant in a certain area. The MESMA definition of SMAs allows for the inclusion of case study areas with different levels of maturity of spatial management plans. Thus the application of the MESMA framework in different areas with different levels of maturity of spatial management plans can consequently lead to different types of assessment outputs. More precisely these outputs can fluctuate from one extreme which is a sustainability appraisal of an existing marine spatial plan, including an 
assessment of the process used to develop the plan, to the other end of the spectrum which reflects an IEA with a set of qualitative recommendations to support an EBM within an SMA context [1].

\subsubsection{The framework steps}

The MESMA framework to evaluate and monitor SMAs comprises seven key steps (Fig. 1) and represents an iterative process [1]. The first step requires the definition of spatial and temporal boundaries to specify the context (step 1a). In relation to those boundaries the high level goals and operational objectives are defined (step 1b). Step 2 comprises the collation of existing information and mapping. In step $2 \mathrm{a}$ the ecosystem components (natural and socio-economic) relevant to the set of objectives are defined and mapped for the SMA. The socioeconomic components (human activities) are mapped and (cumulative) impacts of those on natural ecosystem components are assessed (step 2b). Existing or proposed management measures are further listed in step 2c. Step 3 involves the definition of indicators and related thresholds. Step 4 comprises state assessment of the indicators and/or a risk analysis of management scenarios. Step 5 evaluates the findings against the operational objectives. The evaluation of management effectiveness was conducted in step 6. In step 7 the assessment results were synthesized to formulate recommendations to adapt management.

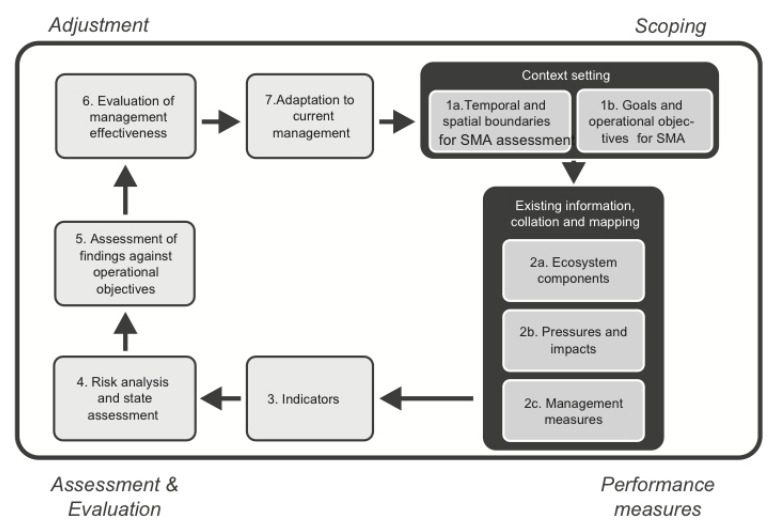

Figure 1: Flowchart with the proposed MESMA framework to monitor and evaluate spatially managed areas (SMAs) through seven key steps (from Stelzenmüller et al. [1]).

\subsection{The study area}

An effort has been made to initiate the process of Ecosystem Based Marine Spatial Management (EB-MSM) [2] in the Greek Ionian Sea and the adjacent gulfs, a region situated in Western Greece (Fig. 2). The study area comprises 10 marine NATURA-2000 sites and includes two national parks: the aforementioned National Marine Park of Zakynthos and the National Park of Messolonghi - Etoliko Lagoons. Part of the study area, especially the semi- 
enclosed Korinthiakos Gulf, has limited connectivity with the open sea, making it vulnerable to intense human activities. Enhanced anthropogenic activities (fisheries, aquaculture, tourism, shipping, and industry) occur along the coasts of the study area and in offshore waters. Growing conflicts exist between human uses and nature conservation as well as among the human uses themselves but there is currently no integrated spatial plan managing the entire study area [3].

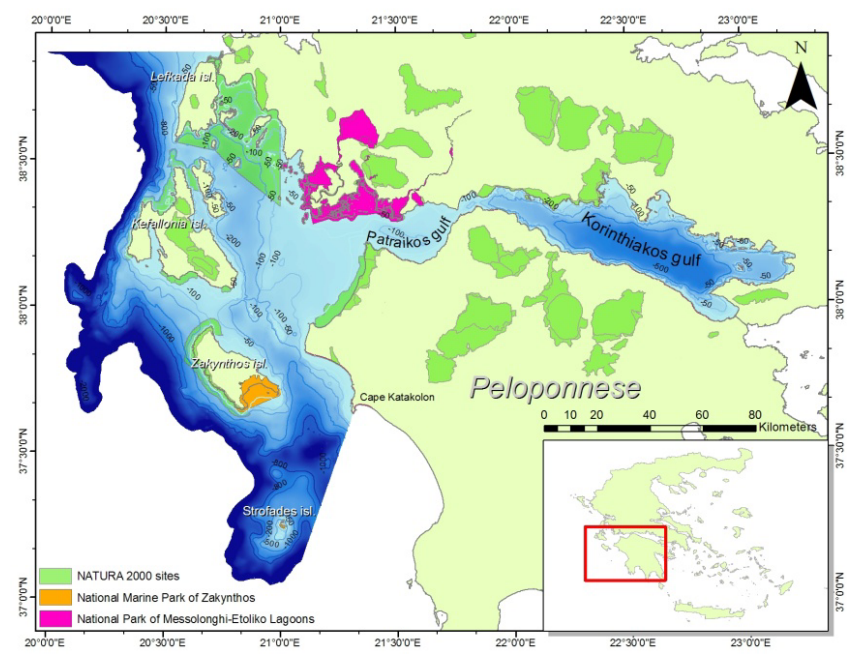

Figure 2: Bathymetric map of the study area with special areas of conservation (from Issaris et al. [3]).

\subsection{Data collection and analysis}

For the application of the MESMA framework in the case study area, several ecosystem components along with selected human activities were mapped. All spatial data were incorporated in a unified geographic information system, using ESRI ArcGIS 9.3 and its various extensions, allowing for a central way of data collection, analysis and presentation.

Additionally, for the needs of further analysis regarding new management proposals, the systematic conservation planning software MARXAN was jointly used, offering solutions for effective decision making while designing Marine Protected Areas (MPAs).

\section{Results}

\subsection{Step 1}

\subsubsection{Spatial boundaries}

The case study boundaries were defined at the very start of the application of the framework based mainly on ecological and geomorphologic criteria, and taking also into account the following points: 
(1) Sectoral initiatives and relevant legislation are in their majority general referring to the entire national marine region and thus would not be decisive in delineating the boundaries of the case study area

(2) Institutional landscape (which will be further analyzed during the Governance Analysis) does not seem to affect the selection of the case study boundaries

(3) Ecological processes and distribution of marine populations (esp. of marine mammals) are not constrained by any human boundaries (national, institutional, jurisdictional etc.)

In the Internal Ionian Archipelago (including adjacent gulfs) most of the boundaries especially in the eastern side and around enclosed gulfs were defined by the coastline (Fig. 2).

The western and southern boundaries were defined by the 2000-m bathymetric contour. As the conservation of the populations of cetaceans in the area are among the priorities of management, and as sperm whales and Cuvier's beaked whales are actively foraging down to $2000 \mathrm{~m}$ depth, we decided to include the entire distributional range of these species in the area by defining the 2000-m depth contour as the natural boundary of the case study region. Southwards we extended the case-study region so that the Strofadia Islands are included, which is a marine protected area of high conservation value.

\subsubsection{High-level goals and operational objectives}

High-level goals were derived from legal obligations concerning various EU Directives, such as the Water Framework Directive (WFD), the Common Fisheries Policy, the Birds Directive 1979/409/EEC - 2009/147/EU, the Habitats Directive 92/43/EEC, the Marine Strategy Framework Directive (MSFD) 2008/56/EU and other legal obligations deriving from the signing of various international agreements, namely the Bern Convention 1979, the RAMSAR Convention 1971, the ICZM Protocol to the Barcelona Convention 2009/89/EU, etc. The respective high-level goals deriving from these obligations are for example the halt of habitat and biodiversity loss, the conservation of wild fauna and flora, the reduction of pollution in the Mediterranean Sea, the Good Ecological Status of regional waters, the long-term sustainable water management based on high level of protection of the aquatic environment, the exploitation of living aquatic resources that provides sustainable economic, environmental and social conditions, etc.

Furthermore, based on the goals mentioned above, specific operational objectives were set, such as: "Ensure sustainability of the population of endangered species Monachus monachus", "Include at least $60 \%$ of priority habitats and species of the Habitats Directive in Natura 2000 network of sites", "Maintain seafloor integrity", "Achieve sustainable exploitation of fishery resources ensuring Good Ecological Status in commercial species", to name a few. Certain socio-economic objectives linked with National (Greek) Master Plans were also identified and were mainly related to coastal development issues (e.g. tourism). All of the operational objectives were found to comply with the 
SMART protocol that is necessary for the effective evaluation of the management performance of spatially managed areas [4].

\subsection{Step 2}

\subsubsection{Collection of existing information and mapping}

Main ecosystem components (environmental and socio-economic) of the study area that were relevant to the operational objectives defined in the previous step were identified and information regarding their spatial distribution and extend was collected and mapped in a GIS system.

Habitat types and species considered in this analysis were selected based on their conservation importance defined by European legislation or international agreements. Habitat types of conservation value include Posidonia oceanica meadows, coastal lagoons, and submarine structures made by leaking gases and reefs. The list of species addressed in the study includes the Mediterranean monk seal Monachus monachus, the common bottlenose dolphin Tursiops truncatus, the stripped dolphin Stenella coeruleoalba, the short-beaked common dolphin Delphinus delphis, the sperm whale Physeter macrocephalus, the Cuvier's beaked whale Ziphius cavirostris, the loggerhead turtle Caretta caretta, the gold coral Savalia savaglia, the short-snouted seahorse Hippocampus hippocampus and the long-snouted seahorse H. guttulatus, the fan mussel Pinna nobilis, the Mediterranean shag Phalacrocorax aristotelis desmarestii, and Cory's shearwater Calonectris diomedea. Among human activities taking place in the study area and analyzed are: fisheries and tourism. Detailed description of data sources, data quality and maps of extend can be found in a recently published study [3]. Herein, a representative map is presented with the distribution and spatial extent of habitat types targeted for conservation (Fig. 3) in the study area.

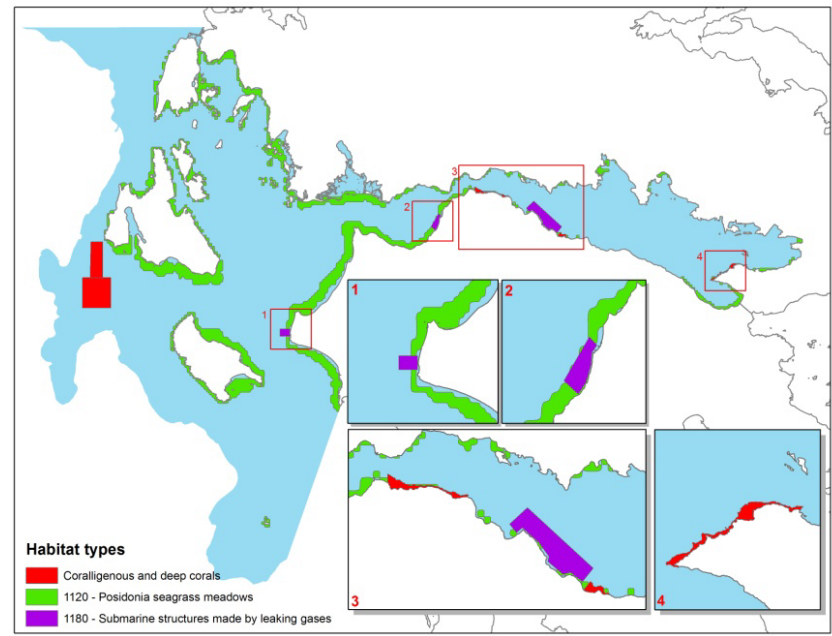

Figure 3: Spatial distribution and extent of habitat types targeted for conservation (from Issaris et al. [3]). 


\subsubsection{Identification of pressures and impacts}

The relationships and possible conflicts of the selected human activities and the ecosystem components were mapped using GIS.

Pressures from trawling activities appear to have the highest impact on certain benthic ecosystem components, such as Posidonia oceanica seagrass beds and cold seep fields (Fig. 4). Concerning the impacts of tourism on the ecosystems components, data are too sporadic and fragmented in order to be measurable and representative. According to findings of the Land Use Simplified Index that was used to quantify pressures from land based activities (urbanization, agriculture, industry) the relevant impacts appeared to range from very low to high [5].

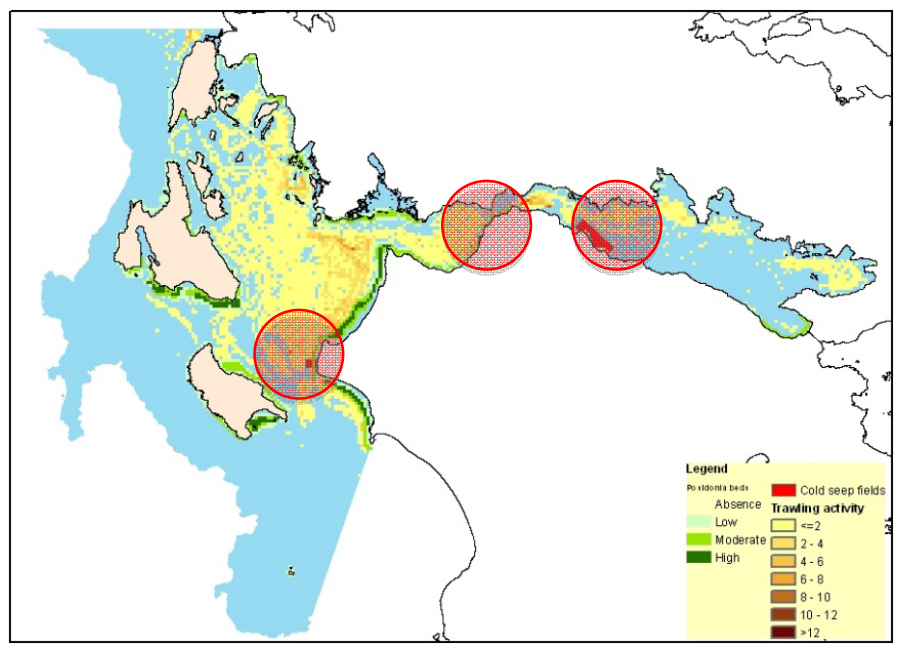

Figure 4: Trawling pressure on benthic ecosystem components (from MESMA Deliverable 3.3 [5]).

\subsection{Step 3: indicators and related thresholds}

Based on the identified operational objectives and their related attributes, a set of indicators was selected to measure the status of each specific attribute. A total of 61 indicators were initially determined [5], most of them based on the proposed indicators by the Commission Decision 201/477/EU on criteria and methodological standards on Good Environmental Status (GES) of marine waters.

Each of these indicators was evaluated and scored based on weighted criteria such as "relevancy to the stated operational objective", "sensitive to manageable human activity", "sensitivity to change", "measurable over a large proportion of the study area". According to their relevant scoring and availability of needed data, 10 indicators, 14 "snapshot" indicators and 13 metrics were finally selected. 


\subsection{Step 4: risk analysis}

The indicators and metrics selected were used to assess the risk of not meeting the operational objectives. For every indicator and metric, the magnitude of the impact of specific type of pressure - as defined in MSFD Annex III and by the MarLIN initiative [6] - was assessed using existing knowledge, while the likelihood of risk occurrence is evaluated compiling results and - in the cases of indicator snapshots and metrics - through expert judgment.

For example, the fisheries related Hake-MSFD 3.2.2 indicator's pressure "Biological disturbance: removal of target species" was found to have a "high" magnitude of impact and a "medium" likelihood of occurrence, while the cetacean related Physeter-MSFD 1.1.1 snapshot indicator's pressure "Biological disturbance: death or injury by collision with ships; death or injury caused by underwater noise" was found to have both "high" magnitude of impact and likelihood of occurrence [5].

Based on this analysis, cumulative assessment of magnitude of impact and likelihood of occurrence resulted in the characterization of the risk of impact -as low, medium, or high- of each of the identified pressures [5]. Since in most cases risk of impact was characterized as "high", there is an indication that the relevant management measures have been inefficient in relation to the objectives set. However, the limited data do not allow the extraction of firm conclusions and outcomes should be taken as purely indicative.

\subsection{Step 5: assessing findings against operational objectives}

Based on the results of the risk analysis success of the defined operational objectives was assessed further. The extent of gap between the current status and the relevant target/threshold was evaluated for all indicators whose data availability allowed reaching a specific conclusion (most snapshot indicators were not included in this process), thus revealing whether the predefined goal of each of the specific operational objectives was either achieved or not.

For example, in the case of the operational objective "Ensure sustainable exploitation of fish stocks", the indicator Hake-MSFD 3.1.1 with the threshold set as equal or less than the Maximum Sustainable Yield of the species, was assessed to have a gap of $17.29 \%$, while for the operational objective "Include at least $60 \%$ of priority habitats and species of the Habitats Directive in Natura 2000 network of sites", the metric Posidonia-PROTECTED with a threshold set at $60 \%$ was found to have a $40 \%$ extent of gap towards meeting its conservational goal.

However, as mentioned before, the limited existing data do not allow the extraction of solid conclusions, highlighting the critical gap in both data availability and knowledge, relevant for conducting thorough assessments that should be tackled particularly via implementing suitable monitoring programs. 


\subsection{Step 6: evaluate management effectiveness}

Management measures appeared to contribute adequately to the achievement of the objectives "Ensure sustainability of the population of the endangered Mediterranean monk seal Monachus monachus" and "The abundance and distributional range of the loggerhead sea turtle Caretta caretta is stable or has a positive trend", possibly due to focused actions such as the establishment of the National Marine Park of Zakynthos, and systematic conservation actions by environmental NGOs mainly through EU funded projects (e.g. LIFE).

In relation to the objectives related to provisions under the Habitats Directive, they appeared to be partly achieved, and pertinent management measures seemed to be in the right direction.

For the objectives related to the MSFD goals no evaluation could be made, since the implementation of the MSFD was still at the Initial Assessment (IA) phase in Greece at the time that this study was undertaken, and following the foreseen process management measures will be taken in 2015.

As for the objectives relevant respectively to "Sustainable tourism development" and "Sustainable coastal development" did not achieve their purpose, and the main reason seemed to be that they are currently tackled through national Master Plans (i.e. The National Master Plans for Tourism, Aquaculture and Urban Development), which are too general in guidelines and provisions and thus cannot be effective in dealing with the specific needs arising from the peculiarities of local conditions.

\subsection{Step 7: recommended adaptations to current management}

As the objectives related to the implementation of the Habitats Directive were considered to be partly achieved, an effort was made to contribute with filling the respective conservation gap by suggesting adaptations to the existing NATURA 2000 network in the area of the Greek case study. Spatial analysis of the features of conservation interest present in the area (based on best available data) was performed and through systematic conservation planning techniques using the MARXAN specialized conservation planning software with consideration of the socio-economic cost - new areas for conservation management were identified (see [7]).

Setting targets of $60 \%$ for high priority conservation features and $20 \%$ for lower priority features and forced the selection of all Natura 2000 sites (scenario A), the best solution accounted for $24 \%\left(3646 \mathrm{~km}^{2}\right)$ of the study region (Fig. 5). Whereas, when we forced the selection of National Parks only (scenario B), the best solution accounted for $18 \%\left(2720 \mathrm{~km}^{2}\right)$ of the total area (Fig. 6). 


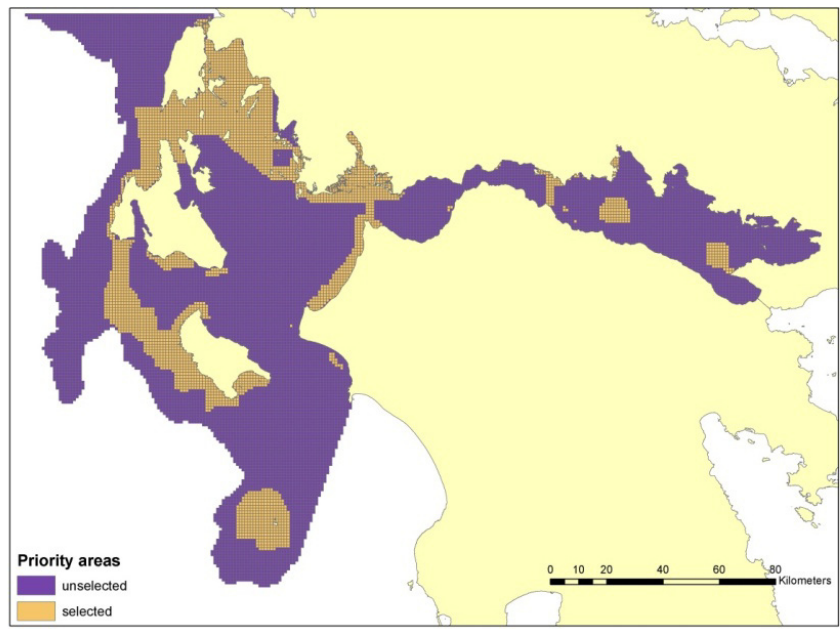

Figure 5: Priority areas selected under scenario A (from Giakoumi et al. [7]).

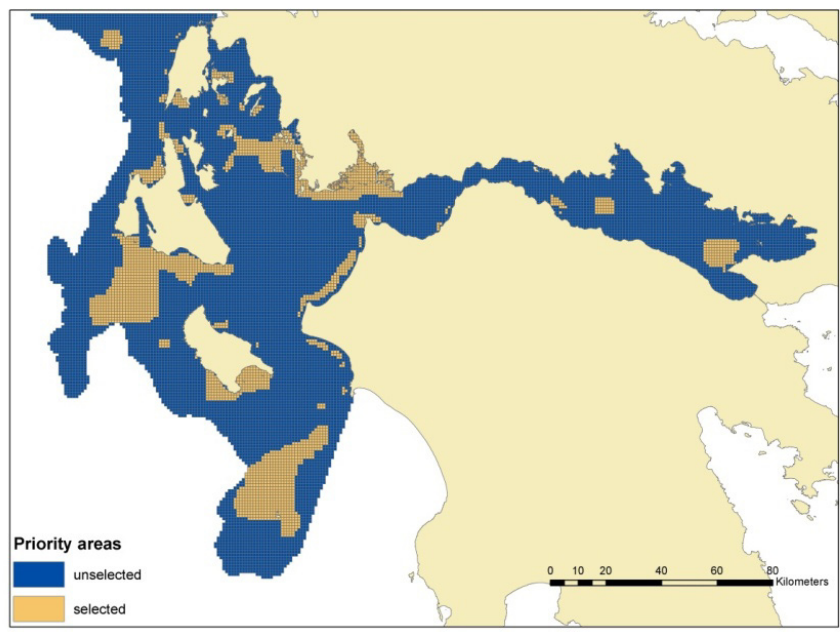

Figure 6: Priority areas selected under scenario B (from Giakoumi et al. [7]).

\section{Conclusions}

The case study area on which the MESMA framework has been applied does not have a holistic management plan, while there are sectoral and geographically scattered spatial management plans, such as the national (Greek) Master plans for touristic and urban development. Growing conflicts exist among human uses like commercial fisheries and tourism, but also between human uses and nature 
conservation. The framework was used to evaluate objectives linked to high level policy goals and certain national sectoral plans, spot data and knowledge gaps, and recommend appropriate initiatives.

An issue that emerged from the initial steps was the lack of high quality data. All analyses were based on the best available data, but quite often surrogates of low spatial and temporal resolution were used. This is highlighted in a recent paper dealing with the data collection process followed in the Greek case study area, and including the respective data quality assessment [3]. The need for higher quality data, both for environmental and socio-economic components, is evident for conducting an integrated monitoring and evaluation of a spatially managed area, and/or for proposing effective management plans.

General outcomes showed that the whole process was informative for both scientists and managers and could provide potential guidelines that could be considered for developing MSPs in the region. Moreover, systematic conservation planning appeared to be a useful tool, which takes also into account the trade-offs involved. Should the designation of the proposed NATURA 2000 sites materialize in the case study area, specific management plans with clear conservation guidelines, rules and penalties for the involved actors should be put in immediate effect [7]. An emerging issue in the latter process appears to be the involvement of key stakeholders who have unique knowledge concerning local features and needs and can provide a better understanding of the potential impact of MSPs on socio-economic sectors [8]; the latter highlights the necessity for bottom-up mechanisms that will ensure high compliance of management measures identified during the process as the most appropriate to be enforced.

\section{References}

[1] Stelzenmüller, V., Breen, P., Stamford, T., Thomsen, F., et al. Monitoring evaluation of spatially managed areas: A generic framework for implementation of ecosystem based marine management and its application. Marine Policy, 37, pp. 149-164, 2013.

[2] Katsanevakis, S., Stelzenmüller, V., et al. Ecosystem-based marine spatial management: Review of concepts, policies, tools and critical issues. Ocean and Coastal Management, 54, pp. 807-820, 2011.

[3] Issaris, Y., Katsanevakis, S., Pantazi, M., Vassilopoulou, V., Panayotidis, P., Kavadas, S., Kokkali, A., Salomidi, M., Frantzis, A., Panou, A., Damalas, D., Klaoudatos, D., Sakelariou, D., Drakopoulou, V., Kyriakidou, C., Maina, I., Fric, J., Smith, C., Giakoumi, S., Karris, G., Ecological mapping and data quality assessment for the needs of ecosystem-based marine spatial management: case study Greek Ionian Sea and the adjacent gulfs. Mediterranean Marine Science, 13(2), pp. 297-311, 2012.

[4] Doran, G. T., There's a S.M.A.R.T. way to write management's goals and objectives. Management Review, 70(11), pp. 35-36, 1981.

[5] MESMA Deliverable 3.3. Applicability of Framework. Part VII: 1st run application of Inner Ionian Archipelago - Patraikos and Korinthiakos Gulfs Case Study. Technical Report, MESMA project (www.mesma.org), 243p., 2011. 
[6] MarLIN initiative, www.marlin.ac.uk/maritimeactivitiesmatrix.php

[7] Giakoumi, S., Katsanevakis, S., Vassilopoulou, V., Panayotidis, P., Kavadas, S., Issaris, Y., Kokkali, A., Frantzis, A., Panou, A., Mavromati, G. Could European marine conservation policy benefit from systematic conservation planning? Aquatic Conservation: Marine and Freshwater Ecosystems, DOI: 10.1002/aqc.2273, 2012.

[8] Vassilopoulou, V., Mavromati, G., Panayotidis, P., Kokkali, A., Anagnostou, C. How effective are Marine Spatial Plans? Framing key issues using stakeholders' opinion. Abstract, ASLO Aquatic Sciences Meeting, 1722 February 2013, New Orleans, Louisiana, USA. 